\title{
Superconductivity two decades on
}

\section{DENVER, COLORADO}

They were lionized in the press, celebrated by the US government, and even given exclusive access to New York City nightclubs - that was 20 years ago. This week, the physicists who made key early breakthroughs in the field of high-temperature superconductivity recalled their fame and expressed hopes that the field would once again dramatically move forward.

Back then, in a marathon session on the evening of 18 March 1987, at a meeting of the American Physical Society, researchers discussed a new-found class of 'superconducting' material that carried electrical current without resistance at dramatically higher temperatures than its predecessors. The impromptu lectures at the New York City Hilton ran until after 3 a.m. and were later dubbed the "Woodstock" of physics. "The euphoria was really unbelievable," says Paul Grant, a physicist at Stanford University, California, who was there that night.

By contrast, the mood was nostalgic at this year's American Physical Society meeting, held this week in Denver, Colorado, when many of those at the original 1987 session gathered for a reunion and to discuss the future.

Superconducting materials differ markedly from other conductors. Normally, the atomic lattice in a metal causes electrons to scatter, creating heat. But in superconductors, the electrons pair together in a way that allows them to travel effortlessly through the material. Superconductivity was first discovered in 1911, and described in theoretical calculations in 1957 by John Bardeen, Leon Cooper and John Robert Schrieffer.

The materials offer the tantalizing possibility of allowing energy to be transmitted with virtually no loss, promising powerful motors and better generators. But the low temperatures at which superconductors originally worked prevented them from being practical. For most of the 1970s and 1980 s, the record for a high-temperature superconductor remained at a miserable 23 kelvin, or 23 degrees above absolute zero.

Then in 1986, Georg Bednorz and Alex Müller of IBM's Zurich Research Laboratory in Rüschlikon discovered a new class of superconductor that worked at $30 \mathrm{~K}$. These cuprates, as they are known, consisted of layers of copper oxide with small amounts of other materials added. The IBM work was soon confirmed by others, including Paul Chu of the University of Houston, Texas, who discovered an

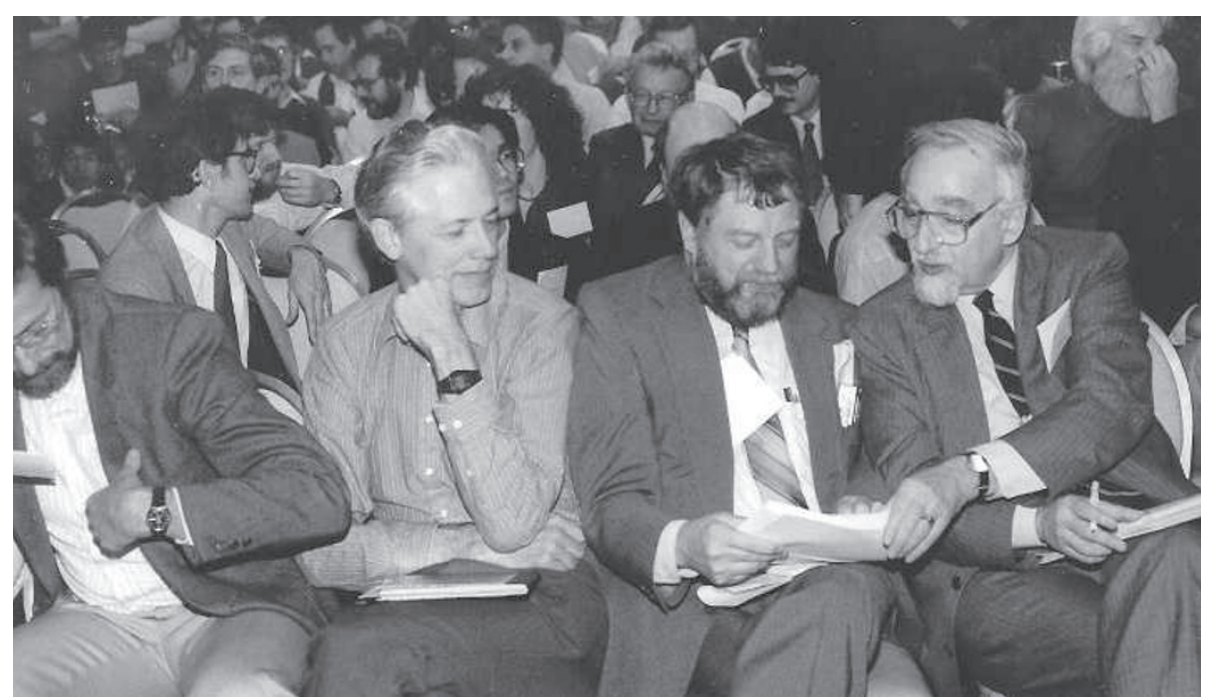

The "Woodstock" of physics: 18 March 1987, when physicists met to discuss a stunning class of material.

yttrium-barium-copper oxide material that could superconduct at $93 \mathrm{~K}$, above the temperature of liquid nitrogen. The discovery meant that superconductors would be cheaper to cool and easier to use. "It created the feeling in many people that nothing is impossible," says Bednorz, who won the 1987 Nobel physics prize with Müller for their discovery.

Condensed-matter physicists found themselves turned into overnight celebrities. "New Superconductors Offer Chance to Do the Impossible," trumpeted The New York Times. Physicists were invited to the White House for a demonstration to then-President Ronald Reagan and given free passes to a New York City discothèque. "It was a heady, heady experience," says Grant. Buoyed by the promises, Congress passed the Superconductivity Competitiveness Act of 1988, which boosted funding for the field.

But there remained a problem. Nobody knew exactly how the new materials worked. After their initial discovery, little progress was made in increasing their operating temperatures. And, because the materials were ceramic, they proved difficult to manufacture and incorporate into existing infrastructures.

Today, says Grant, little has come of those early claims. "No high-temperature superconducting technology is turning a profit," he says. "If you reflect back on 1987 there were a lot of futures promised, I think irresponsibly."

And the scientific field has also slowed. Although more than 100 superconducting cuprate materials have been discovered, the record for superconductivity remains at $164 \mathrm{~K}$, about halfway between absolute zero and room temperature. The theoretical understanding of the material is incomplete as well, says physicist Douglas Scalapino of the University of California, Santa Barbara. Although many researchers believe that electrons pairs underlie superconductivity even in these new materials, no one knows how the electrons bind together. "We don't understand what causes it," he says.

A low point came last September when two German researchers predicted that, given current trends, publications of papers in high-temperature superconductivity would end between 2010 and 2015. The researchers later revised their estimates, but not before angering many scientists (see Nature 443, 376-377; 2006).

But despite the frustrating lack of progress, many remain hopeful. Room-temperature superconductors may yet be found, says Chu: "At this moment, there's no evidence, either theoretical or experimental, that tells us it's impossible."

And high-temperature cuprates may soon find some applications in defence, says Grant. The materials' electrical efficiency may make them useful for high-power microwave beams that can be used to disable small ships.

Optimists say the field could be reinvigorated at any time by a new discovery, whether through serendipity or otherwise. "If something happens, there will be another Woodstock and another explosion," Grant says. Geoff Brumfiel 